\title{
Endothelium-dependent relaxation induced by etomidate in the aortas of insulin-resistant rats
}

\author{
Wenxin Xue ${ }^{1}$, Yiwen $\mathrm{Li}^{1}$, Jing $\mathrm{Li}^{1}$, Li Yan² ${ }^{2}$ Fang Yang ${ }^{3}$
}

${ }^{1}$ Department of Pharmacy, Meitan General Hospital, Beijing, China

2Department of Physiology and Pathophysiology, Institute of Basic Medical Sciences, Chinese Academy of Medical Sciences and Peking Union Medical College, Beijing, China

${ }^{3}$ Department of Neurology, Meitan General Hospital, Beijing, China

Submitted: 25 August 2016

Accepted: 15 December 2016

Arch Med Sci 2018; 14, 5: 1155-1162

DOI: https://doi.org/10.5114/aoms.2018.77256

Copyright $\odot 2018$ Termedia \& Banach

\section{A bstract}

Introduction: Few reports have mentioned the effect of etomidate on the aortas of insulin-resistant (IR) rats. In this study, we investigated the effect of etomidate on isolated IR aortas of rats, and explored its underlying mechanism.

Material and methods: The IR rat model was established through feeding with a high-fructose diet. The systolic blood pressure (SBP) was measured by the tailcuff method before grouping and at the end of the 8-week feeding; blood samples were also obtained for analysis. Thoracic aorta rings of IR rats were isolated and suspended in a tissue bath. The tensile force was recorded isometrically. The effect of etomidate on provoked contraction of the rings was assessed with or without a potassium channel blocker or NO synthase inhibitor.

Results: Etomidate-induced relaxation in IR rings was greater than normal control (NC) rings (all $p<0.001$ with etomidate log $M$ of -4 to -6 ). NG-nitro-L-arginine methyl ester (L-NAME, an NO synthase inhibitors) inhibited etomidate-induced relaxation in NC rings, but had no effect on the IR rings (all $p<0.001$ with etomidate $\log \mathrm{M}$ of -4 to -6 ). Pre-incubation with glibenclamide (Gli, a potassium channel blocker) significantly inhibited etomidate-induced relaxation in NC and IR rings (all $p<0.001$ with etomidate $\log M$ of -4 to -6 ), and had no inhibited effect on endothelial denuded aortic rings. Conclusions: Insulin resistance increased etomidate-induced relaxation in rat aortas. Etomidate causes vasodilation in IR rat aortas via both endothelium-dependent and independent ways; impaired NO-mediated relaxation was disrupted and ATP-sensitive potassium $\left(\mathrm{K}_{\mathrm{ATP}}\right)$ channel-mediated relaxation may be involved in the endothelium-dependent relaxation of etomidate in IR rats.

Key words: insulin resistance, etomidate, vascular relaxation, endothelial function, ATP-sensitive potassium $\left(K_{\text {ATP }}\right)$ channel.

\section{Introduction}

Insulin resistance (IR) is a physiological condition in which cells have lower sensitivity or a reduced response to insulin. Insulin resistance is a risk factor associated with cardiovascular diseases including hypertension, dyslipidemia, type 2 diabetes, and obesity $[1,2]$. Insulin resistance has profound negative effects on artery function throughout the body [3]. Studies have demonstrated that IR impairs vascular function of the aorta and coronary artery [4, 5], mesenteric function and cerebral vascular beds [6]. Recent studies showed that IR is not only associated with the occurrence

\author{
Corresponding author: \\ Fang Yang \\ Department of Neurology \\ Meitan General Hospital \\ Xibahenanli 29 \\ Chaoyang District \\ Beijing 100028, China \\ Phone: +86 1084512685 \\ Fax: +86 1064667755 \\ E-mail: fangyoung1990@163. \\ com
}


and development of vascular lesions, but is also associated with the surgical stress response and anesthesia safety. Insulin resistance patients have poor tolerance of surgery. In addition, the anesthetic pharmacokinetic characteristics of IR patients are also different from those of non-IR patients.

The increasing number of patients with diabetes mellitus (DM) makes the work of the anesthetist even more challenging during the perioperative period: hyperglycemia might occur in the perioperative period due to a combination of tissue insulin resistance and decreased insulin secretion [7]. In addition, general anesthesia might cause a dangerous increase or decrease of systemic or pulmonary pressure because of variations in heart rate and pulmonary flows. Such variations during catheterizing would make the procedure useless. Exploring the effects of anesthetics on vascular responsiveness could provide useful information for anesthesia of IR patients. Etomidate is an anesthetic often used in inpatients with valvular or ischemic heart disease. Etomidate does not inhibit sympathetic tone or myocardial function. Typical anesthesia induction doses of etomidate induce only minimal changes in blood pressure and heart rate [8]. Ouedraogo et al. [9] reported that etomidate exerts relaxant effects on the pulmonary artery (PA) in chronically hypoxic $(\mathrm{CH})$ rats, and etomidate could inhibit $\mathrm{K}_{\text {ATP }}$-mediated pulmonary vasorelaxation via a direct effect on pulmonary vascular smooth muscle [10].

Endothelium regulates vasoconstriction through releasing vasodilating and vasoconstriction substances. Compelling evidence demonstrated the parallel progression between insulin resistance and endothelial dysfunction [11-13]. Bornfeldt et al. [14] induced endothelial cell (EC) insulin resistance using the Cre-loxP method in a non-atherosclerotic model, in which ECs and aortas showed reduced levels of eNOS and endothelin-1 mRNA. Chronic consumption of diets with high fat and high sugar causes several abnormal characteristics of metabolic syndrome, including insulin resistance, obesity, and hypertension in rodents $[15,16]$.

We hypothesized that the effect of etomidate in IR rat aortas was associated with endothelium-derived mediators such as NO and/or others. To explore the mechanism of the etomidate-mediated relaxation on the thoracic isolated IR aortas of rats, we evaluated the effect of etomidate on phenylephrine (PE)-induced contraction [17], and etomidate on endothelium-dependent relaxation in rat aortas interfered with by different drugs [18].

\section{Material and methods}

\section{Animal and ethical considerations}

All procedures and experimental protocols were carried out in accordance with EU Directive
2010/63/EU for animal experiments. Thoracic arteries were taken from male Sprague-Dawley rats (3-4 months, supplied by the Animal Center of Shanxi Medical University).

After feeding for 2 weeks with the normal diet, the rats were randomly assigned to the control diet group (NC, continued to be fed with normal control diet) or the fructose diet group (IR, fed with a diet containing $60 \%$ fructose, $11 \%$ fat, and $29 \%$ protein for 8 additional weeks). All rats were given free access to food and water and maintained in a 12-h light/dark cycle. Rats were housed in cages to collect 24-h urine samples and the food and water consumption were recorded. The systolic blood pressure (SBP) was measured by a tail-cuff method before grouping and the end of the 8-week period; the blood samples were also obtained for analysis.

\section{Drugs and regents}

Etomidate was purchased from Nhwa (Nhwa Pharmaceutical, Jiangsu, CHN). Intralipid was purchased from SSPC (Sino-Swed Pharmaceutical, Jiangsu, CHN). Phenylephrine hydrochloride $(\mathrm{PE}), \mathrm{KCl}$, acetylcholine chloride (ACh), sodium nitroprusside (SNP), L-NAME, indomethacin (Indo), iberiotoxin, 4-aminopyridine (4-AP) and glibenclamide (Gli) were purchased from Sigma (St. Louis, MO, USA). Gli were dissolved in ethanol (final concentration of ethanol in a tissue bath $\leq 0.5 \%$, with no influence on PE-induced contraction). Phenylephrine hydrochloride, etomidate, ACh, SNP, 4-AP and L-NAME were dissolved in distilled water. Indo was dissolved in $4 \%$ (wt/vol) $\mathrm{NaHCO}_{3}$.

\section{Systolic blood pressure measurement}

Systolic blood pressure (SBP) was measured by the tail-cuff method with an electrosphygmograph (PE-300, Narco Bio-Systems, Austin, TX, USA). Before the initiation of fructose feeding, rats were trained and accustomed to daily measurement of SBP by the tail-cuff method. Briefly, rats were placed (9 a.m.) in their maintenance cages in a room at $28^{\circ} \mathrm{C}$ for $2 \mathrm{~h}$; SBP was measured in unrestrained animals. Once the rats were considered not susceptible to stress from the tail-cuff procedure, SBP measurements were performed. SBP was measured on 2 consecutive days at the same time (11 a.m.). Measurements were carried out 8 times in each rat per day; the maximum and the minimum values were rejected.

\section{Biochemical measurements}

Serum insulin levels were measured by radioimmunoassay method. Blood glucose levels were determined by the colorimetric analysis method (Roche Hitachi 717 Chemistry Analyzer, GMI, Inc. USA). Insulin sensitivity index (ISI) is calculated by 
the formula $|S|=-\ln (F B S \times$ fasting serum insulin (FSI); when ISI $\leq-4.88$, the IR model is considered as successful.

\section{Preparation of aortic rings for tension measurement}

After 8 weeks of fructose diet feeding, rats were sacrificed by cervical dislocation then exsanguinated. The thoracic aorta was isolated and transferred into HEPES solution ( $\mathrm{pH} 7.4,4^{\circ} \mathrm{C}$ ) immediately with the following components: $\mathrm{NaCl} 144 \mathrm{mM}, \mathrm{KCl}$ $5.8 \mathrm{mM}, \mathrm{CaCl}_{2} 2.5 \mathrm{mM}, \mathrm{MgCl}_{2} 1.2 \mathrm{mM}$, HEPES $5 \mathrm{mM}$, and D-glucose $11.0 \mathrm{mM}$. The aortas were cut into rings with $3 \mathrm{~mm}$ length. The rings were suspended on 2 wire hooks in a water-bath tube containing HEPES solution. $95 \% \mathrm{O}_{2}$ and $5 \% \mathrm{CO}_{2}$ mixed gas was maintained in the tube at $37^{\circ} \mathrm{C}$. The upper hook was connected with a force transducer, and the changes in isometric force were recorded by PowerLab Chart 5.4 system (AD Instruments, Bella Vista, New South Wale, AUS). The lower hook was fixed. Each ring was equilibrated in $10 \mathrm{ml}$ of bathing solution for $120 \mathrm{~min}$ before the experiment. The resting tension was adjusted to $2 \mathrm{~g}$ (this preload was found to be optimal for force development in preliminary studies).

After the preparatory work, the rings were exposed to $\mathrm{KCl}$ solution $(120 \mathrm{mM})$. Vasoconstriction was terminated $30 \mathrm{~min}$ after equilibration [19]. The rings were rinsed with fresh HEPES solution and allowed to equilibrate for an additional $60 \mathrm{~min}$ before the application of phenylephrine (PE) $(10 \mu \mathrm{M})$. The rings were activated 2 times with $\mathrm{KCl}(120 \mathrm{mM})$ or PE $(10 \mu \mathrm{M})$. The integrity of the endothelium was presumed by the observation that the relaxation induced by acetylcholine (ACh, $10 \mu \mathrm{M})$ on the contraction was greater than $70 \%$. When the contraction induced by PE and the relaxation induced by $\mathrm{ACh}$ were reproducible, the effects of the drugs were tested.

To assess the role of the endothelium in the vascular response of etomidate, endothelium was deprived in some thoracic aortic rings through rubbing of the luminal surface with a string. The removal of the endothelium was tested by the relaxation induced by ACh $(10 \mu \mathrm{M})$ on the contraction being less than $10 \%$. For the relaxation studies, the submaximal contraction was provoked with PE $(1 \mu \mathrm{M})$. When the contraction was steady (30 min after equilibration), etomidate or ACh was added cumulatively to the tissue bath. Response results were expressed as the tension change percentage on PE-induced pre-contraction.

\section{Endothelium and the effect of etomidate}

To determine the extent of endothelium-dependent vasodilation, the following drugs were used for measurement of tension in endothelial-intact and denuded arteries after $\mathrm{PE}$-induced pre-contraction: 1) etomidate alone $\left(10^{-6}\right.$ to $\left.10^{-4} \mathrm{M}\right)$; 2) lipid emulsion (Intralipid, 20\%, the vehicle control for etomidate, $10^{-6}$ to $\left.10^{-4} \mathrm{M}\right)$ and 3$) \mathrm{ACh}\left(10^{-9}\right.$ to $10^{-5} \mathrm{M}$ ) following $10 \mathrm{~min}$ pretreatment with and without etomidate $\left(5 \times 10^{-6}\right.$ and $\left.5 \times 10^{-5} \mathrm{M}\right)$. The etomidate doses used in this study are referenced from other research [18].

Nitric oxide synthase (NOS) inhibitor, epoxy synthase inhibitor and potassium channel blockers and the effect of etomidate

L-NAME, a nitric oxide synthase inhibitor, and indomethacin (Indo), an epoxy synthase inhibitor, were also used to evaluate the interaction effect. L-NAME or Indo was added to the tissue bath $10 \mathrm{~min}$ before different concentrations of etomidate were added. To determine the possible effects of $\mathrm{K}^{+}$channels on the action of etomidate, potassium channel blockers, Gli (10 $\mu \mathrm{mol} / \mathrm{l})$, 4-AP (1 mmol/l), $\mathrm{BaCl}_{2}(1 \mathrm{mmol} / \mathrm{l})$ and iberiotoxin $(100 \mathrm{nmol} / \mathrm{l})$ were added to the tissue bath $10 \mathrm{~min}$ before etomidate was added.

\section{Statistical analysis}

Numerical values were expressed as mean \pm SEM. Vasorelaxation-response results were expressed as the percentage relaxation of the pre-contraction induced by $P E$. The maximum relaxant response (Rmax) was determined as Rmax $=100 \%$, which indicates complete reversal of PE contraction. Statistical analysis was performed using Student's $t$ test for unpaired comparisons. value of $p<0.05$ was considered as statistically significant.

\section{Results}

\section{Laboratory metabolic parameters}

After 8 weeks of feeding, the SBP, fasting blood glucose (FBG), serum insulin, and ISI in the IR group all increased significantly compared with normal controls (Table I, $p<0.05$ ). The insulin resistant rats were successfully cultured.

\section{Effects of etomidate on aortic rings pre-contracted by $\mathrm{PE}$}

The relaxation of etomidate to endothelial-intact and denuded aortic rings in response to $\mathrm{PE}$ contraction were compared in normal control (NC) and IR rats. The tension increments induced by PE $(1 \mu \mathrm{M})$ in IR and NC rings with intact endothelium were $2.13 \pm 0.42 \mathrm{~g}$ and $1.95 \pm 0.38 \mathrm{~g}$, respectively. Etomidate and Intralipid with different concentrations induced different relaxations (Figure 1). The induced relaxation increased with the increasing concentration. The induced relaxations 
Table I. Effects of high-fructose diet on SBP, FBS, FSI, ISI and TG of SD rats $(n=8)$

\begin{tabular}{|c|c|c|c|c|c|}
\hline Group & SBP [mm Hg] & $\mathrm{FBS}[\mathrm{mm}]$ & $\mathrm{FSI}[\mathrm{mU} / \mathrm{l}]$ & ISI $[-\ln (F B S \times F S I)]$ & TG $[\mathrm{mm}]$ \\
\hline NC & $110.41 \pm 1.57$ & $2.79 \pm 0.35$ & $19.15 \pm 3.12$ & $-3.98 \pm 0.07$ & $0.88 \pm 0.17$ \\
\hline IR & $149.2 \pm 1.21^{\star \star}$ & $5.47 \pm 1.38^{\star \star}$ & $30.78 \pm 4.31^{\star *}$ & $-5.12 \pm 0.25^{\star \star}$ & $2.55 \pm 0.21^{*}$ \\
\hline
\end{tabular}

in endothelial-intact rings were higher than endothelial-denuded rings at the same NC $(24.18 \%$ in endothelial-intact ring and $16.66 \%$ in endothelial-denuded ring at the highest concentration of Intralipid, $10^{-4} \mathrm{~mol} / \mathrm{l}$, log M-4) or IR (95.15\% in endothelial-intact ring and $86.48 \%$ in endothelial-denuded ring at the highest concentration of etomidate, log M-4) rings. In addition, the induced relaxations in endothelial-intact rings treated with etomidate were higher than endothelial-denuded rings (both NC and IR rings, log $M-5.5$ to $\log M-4$ concentration of etomidate, $p<0.01$, Figure 1 )

Insulin resistance induced higher relaxations in aortic rings treated with etomidate whether the endothelial was intact or denuded: the induced relaxation were $95.15 \pm 3.09$ in endothelial-intact IR rings and $86.49 \pm 2.23 \%$ in endothelial-denuded IR rings at the highest concentration of etomidate (log M-4). Comparison of results also showed that IR induced higher relaxation than NC arteries

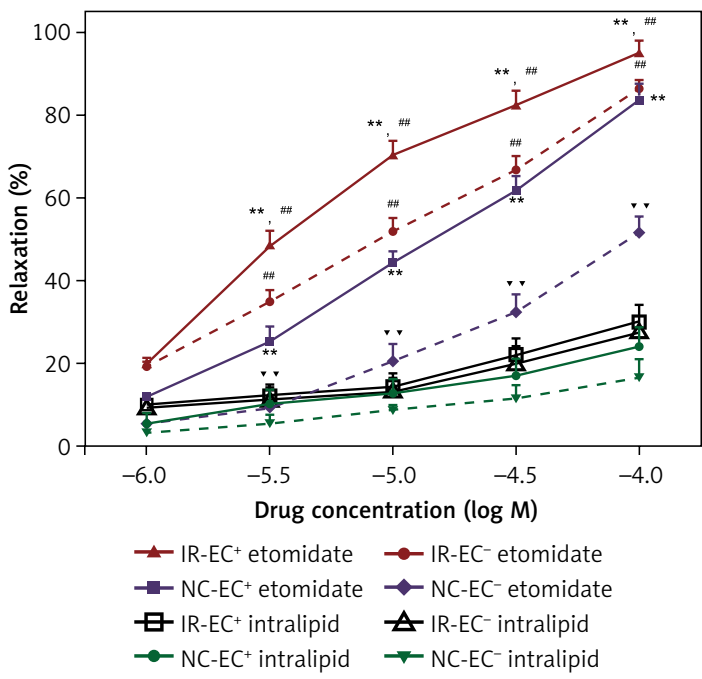

Figure 1. Induced relaxation on pre-contracted aortic rings isolated from NC and IR rats. IR-EC+ etomidate: insulin resistance (IR) ring with intact endothelial $\left(\mathrm{EC}^{+}\right)$treated with etomidate. $\mathrm{NC}$ - normal control. EC- - endothelial denuded. Intralipid: the vehicle control for etomidate

${ }^{* *} p<0.01$ compared with same concentration of etomidate-treated endothelial-denuded rings of IR (or) NC arteries. ${ }^{\# \# p} p 0.01$ compared with same concentration of etomidate-treated endothelial-intact (or denuded) NC rings. $\mathbf{}$ Compared with same concentration of Intralipidtreated endothelial-intact NC rings. All data lines have been listed according to relaxation sequence from high to low.
( $p<0.01$ ) in the same etomidate treated rings when endothelium was intact (or denuded).

\section{Effects of potassium channel blockers on the action of etomidate}

Pre-incubation with 4-AP, Iber, or $\mathrm{BaCl}_{2}$ had no effect on etomidate treated NC or IR rings (Figure 2 A). Pre-incubation with Gli significantly inhibited etomidate-induced relaxation in NC and IR rings (Figure $2 \mathrm{~A})(\log M-5.5$ to $\log M-4$ concentration of etomidate, both $p<0.01$ ); while Gli had almost no inhibitory effect on endothelial-denuded rings of $\mathrm{NC}$ or IR rats (Figure $2 \mathrm{~B}$ ).

\section{Effects of L-NAME and indomethacin on the action of etomidate}

Pre-incubation with L-NAME (0.1 mM) had no relaxation effect on $\mathrm{IR}$ rings, but pre-incubation with L-NAME significantly inhibited etomidate-induced relaxation in NC rings ( $\log M-5.5$ to $\log M-4$ concentration of etomidate, $p<0.01$, Figure 3). Pre-incubation with indomethacin (Indo, $10 \mu \mathrm{M}$ ) had no relaxation effect on NC or IR rings (Figure 3).

\section{Effects of etomidate on ACh-induced relaxation in rat thoracic aorta}

Etomidate significantly inhibited ACh-induced relaxation in NC rings. We observed significantly reduced relaxation caused by etomidate in ACh induced NC rings (maximal relaxation of $84.51 \%$ in NC ring, and $70.1 \%$ and $65.45 \%$ at $5 \times 10^{-6}$ and $5 \times 10^{-5} \mathrm{M}$ of etomidate $\left(3 \times 10^{-7}\right.$ to $3 \times 10^{-5}$ concentration of Ach, both $p<0.01$, Figure 4$)$. In the aortic rings of IR rats, pre-incubation with different doses of etomidate had no significant effect on the relaxation caused by $\mathrm{ACh}$. The ACh-induced relaxation had significant difference in IR rings whether etomidate treated or untreated when compared with NC untreated rings $\left(3 \times 10^{-8}\right.$ to $3 \times 10^{-5}$ concentration of Ach, $p<0.01$, Figure 4).

\section{Discussion}

Etomidate is an imidazole derivative nonbarbiturate intravenous short acting anesthetic. It has no analgesic effect and is especially suitable for patients with shock, cardiovascular risk factors, hemodynamic instability, low blood volume and high airway reactivity. The GABA $A$ receptors are 
A

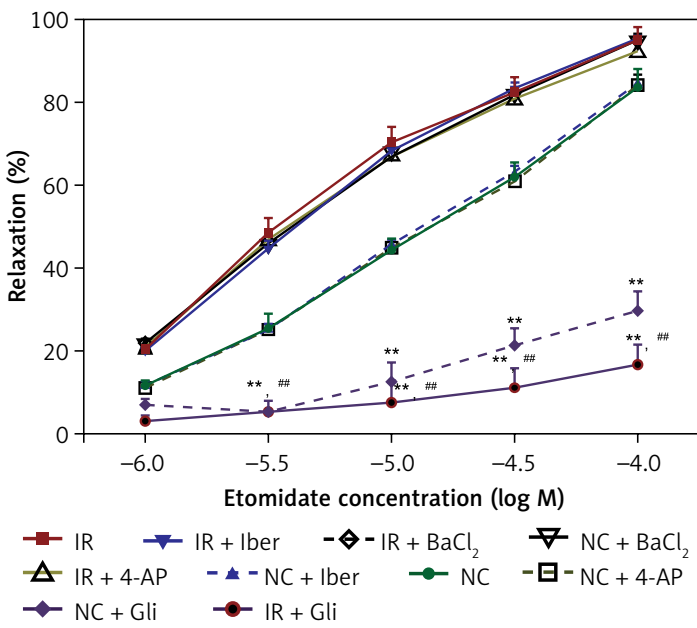

B

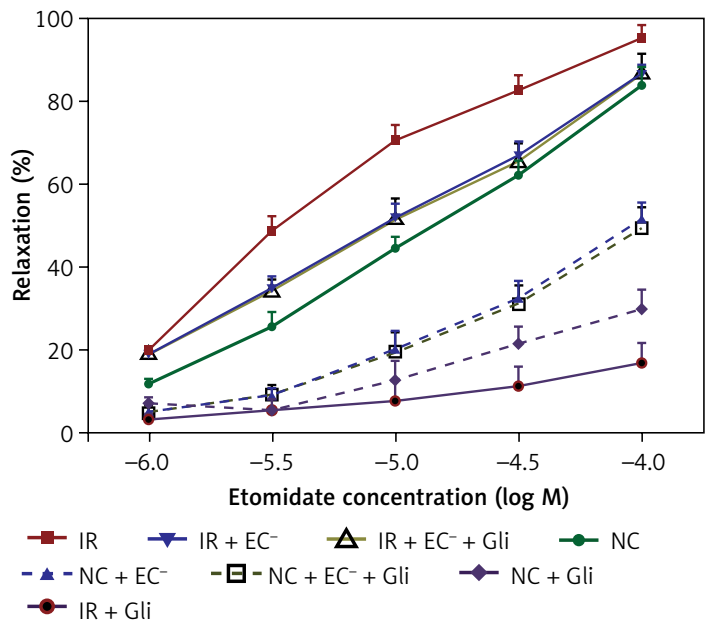

Figure 2. Etomidate-induced relaxation on pre-contracted aortic rings with or without $\mathrm{K}+$ channel inhibitors. A - Pre-incubation with Iber, 4-AP and $\mathrm{BaCl}_{2}$ had no effect on etomidate-treated NC or IR rings. Pre-incubation with Gli significantly inhibited etomidate-induced relaxation in NC and IR rings. B - Gli had no inhibitory effect on endothelial-denuded rings in NC or IR rats

${ }^{* *} p<0.01$ compared with same concentration of etomidate-treated NC rings. ${ }^{\# \#} p<0.01$ compared with same concentration of etomidate-treated IR rings. All data lines have been listed according to relaxation sequence from high to low.

closely involved in the molecular mechanism and regulatory effect of etomidate [20]. Etomidate is also believed to cause generalized vascular relaxation. Shin et al. [17] reported that etomidate attenuates PE-induced contraction in rat aorta. There have been studies indicating that etomidate could inhibit vasorelaxation in canine pulmonary artery [18]. Kessler et al. [21] indicated that the relaxant response of human renal artery to acetylcholine was markedly reduced by the $\mathrm{K}+\mathrm{Ca}$ channel antagonist and the cytochrome P450 inhibitor. They concluded that etomidate selectively attenuated

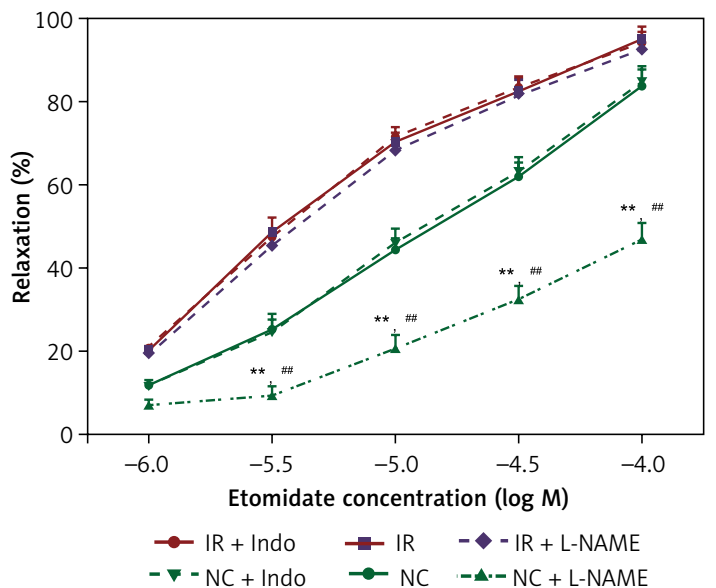

Figure 3. Pre-incubation with indomethacin (Indo, $10 \mu \mathrm{M})$ had no relaxation effect on NC or IR rings; pre-incubation with L-NAME significantly inhibited etomidate-induced relaxation in NC rings

${ }^{* *} p<0.01$ compared with same concentration of etomidate-treated NC ring. \#p $<0.01$ compared with same concentration of etomidate-treated IR rings. All data lines have been listed according to relaxation sequence from high to low. the relaxant response to acetylcholine, and $\mathrm{K}+\mathrm{Ca}$ channels might be involved in the action of etomidate. All these studies focused on the vascular effects of etomidate in normal blood vessels while few studies investigated the effect of etomidate in IR blood vessels. In this study, we found that etomidate could enhance aortic vasodilation of IR rings compared with NC rings. The present study also provides insight as to why enhanced vasodilation occurs in IR arteries at the vascular level.

The doses of etomidate used in the study are clinically relevant. It has been estimated that the

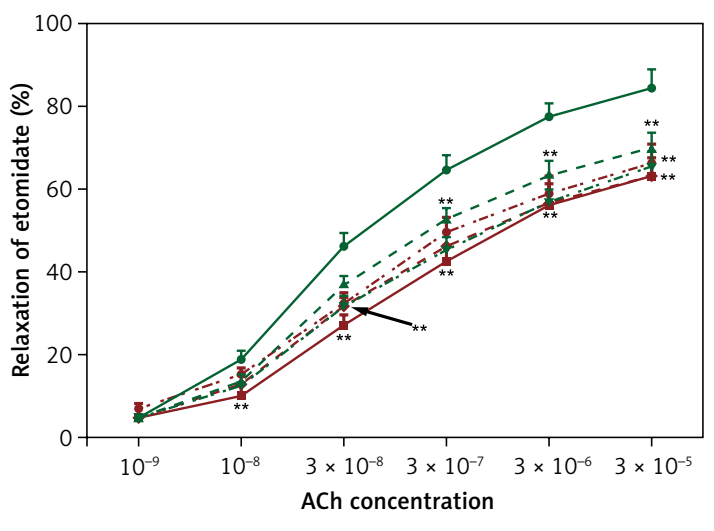

$\rightarrow$ NC $\quad$ - - NC-etomidate $5 \times 10^{-6} \mathrm{~mol} / \mathrm{l}$

- $\mathbf{\nabla}$ - NC-etomidate $5 \times 10^{-5} \mathrm{~mol} / \mathrm{l} \longrightarrow$ IR-etomidate $5 \times 10^{-6} \mathrm{~mol} / \mathrm{l}$

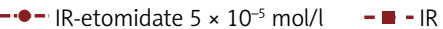

Figure 4. Etomidate significantly inhibited ACh-induced relaxation in NC rings. The ACh-induced relaxation had a significant difference in $I R$ rings whether etomidate treated or untreated when compared with NC untreated rings

${ }^{* *} p<0.01$ compared with same concentration of AChtreated NC rings. 
peak plasma concentration of etomidate during induction of general anesthesia is approximately $10^{-5} \mathrm{M}[22-24]$. The free plasma concentration is likely to be $2.5 \times 10^{-6} \mathrm{M}$. In addition, it has been reported that $5 \times 10^{-5} \mathrm{M}$ concentration of etomidate is clinically relevant [17]. Etomidate could cause relaxation in rat aorta. It is interesting that the Intralipid vehicle also induces slight relaxation, which is greater in intact arteries than denuded arteries. This suggests that denudation removes endothelium-derived vasodilators, which normally antagonize the constriction caused by PE, or Intralipid causes endothelium-dependent vasodilation. Indeed, it has been suggested that Intralipid itself interacts with the NO pathway [25]. However, etomidate induced significantly higher vasodilation than Intralipid, and IR will expand the vasodilation. It is more reasonable to infer that the vasodilation of Intralipid is due to the stimulation of blood vessels.

Endothelial cells contribute to the local regulation of vasomotor tone by releasing dilator and constrictor substances. The endothelium plays a crucial role in regulating the tone of vascular smooth muscle and vasoconstriction via different substances [26, 27]. Nitric oxide and prostacyclin are endothelium-derived mediators [28]. In addition, the endothelium can release endothelium-derived hyperpolarizing factors (EDHFs) [29]. There are many candidate EDHFs, including $\mathrm{H}_{2} \mathrm{O}_{2}$ [30], and the $\mathrm{K}^{+}$iron [31]. It is notable that EDHF has species- and vascular bed specific character, and none of them is a universal factor [31]. Sohn et al. [18] indicated that etomidate at clinically relevant concentrations attenuates endothelium-dependent relaxation induced by acetylcholine by acting at a site distal to the endothelial muscarinic receptor. Edwards et al. [32] demonstrated that $\mathrm{K}+$ serves as an EDHF in the mesenteric artery by activating the $\mathrm{K}$ channel. It is reported that the vasorelaxation induced by etomidate was caused by the activation of KATP channels in canine pulmonary arteries [10] and $\mathrm{K}+\mathrm{Ca}$ channels in human renal arteries [21]. Collectively, these reports suggest that etomidate relaxes arteries via multiple and complex mechanisms, which may vary in different tissues.

In our study, denudation of the endothelium reduced etomidate-induced relaxation but did not abolish it completely; this suggests that etomidate causes relaxation via both endothelium-dependent and endothelium-independent ways. Potassium $(\mathrm{K}+)$ channels or leak channels are major channels influencing neuronal membrane potential and excitability, and have many important physiological functions when participating in the process of anesthesia. Our findings indicate that Gli inhibits etomidate-induced relaxation in both NC and IR aortic rings in an endothelium-depen- dent way, while 4-AP, iberiotoxin, and $\mathrm{BaCl}_{2}$, the other 3 potassium channel blockers, showed no obvious effects on the relaxation, which suggests that the induction effect of etomidate partially relies on $\mathrm{K}_{\text {ATP }}$ channels from the endothelium but not other K+ channels.

Deficiency of endothelium-derived NO is believed to be the primary defect that links insulin resistance and endothelial dysfunction [33]. We found that pre-incubation with L-NAME, an NO synthase inhibitor, had no relaxation effect on $\mathrm{IR}$ rings, but had an inhibitory effect on etomidate-induced relaxation in NC rings. Our results support this conclusion: relaxation of the aorta induced by etomidate was related to the synthesis of NO in normal rats. A possible explanation is that etomidate may increase NO synthesis and the bioavailability of NO in normal rats, while under the conditions of IR, the NO pathway is disrupted. Ogawa et al. [34] indicated that propofol inhibited the synthesis of prostacyclin in rabbit mesenteric resistance arteries. Horibe et al. [35] demonstrated that prostacyclin did not participate in ACh-induced pulmonary vasorelaxation. We did not find that etomidate has a relaxation effect on indomethacin treated IR rings. This suggests that the arachidonic acid-cyclooxygenase pathway was not involved in the etomidate-induced relaxation in $\mathrm{IR}$ aorta and the precise mechanism of the effect of etomidate in IR is unclear and needs further research.

It is believed that acetylcholine (ACh) could dilate normal blood vessels by promoting the release of a vasorelaxant substance from the endothelium, while it constricts blood vessels if the endothelium is removed experimentally [36]. Oyama et al. [37] reported that insulin resistance led to acetylcholine-induced microvascular constriction in a patient with vasospastic angina. Consistent with Oyama's report, our research demonstrates that insulin resistance attenuates the relaxation caused by Ach, and administration of etomidate could partially reverse IR-induced vasoconstriction in Ach treated aorta. Another interesting finding in our study was that the increase of ACh-induced dilation by etomidate in IR rings did not have the same effect compared with ACh-induced NC rings treated with etomidate. Previous studies have indicated that as a result of endothelial dysfunction, activation of $\mathrm{K}_{\text {ATP }}$ channels is impaired in IR rats [38]. Therefore, we speculate that etomidate's effect on ACh-induced constriction in IR aorta may be associated with impaired activation of $\mathrm{K}_{\text {ATP }}$ channels.

There are some deficiencies of our study: firstly, the effect of insulin resistance on different parts of the blood vessels may be different [39]. The study of etomidate in more parts of the vascular such as the coronary artery and renal artery is necessary. Secondly, the isolated environment is not equiv- 
alent to the body; in addition to the changes in vascular tension, the body's environment is also more complex. Hence, further studies are needed to confirm our preliminary conclusions.

In conclusion, etomidate causes relaxation in normal and IR aortas rings, and the relaxation is much higher in IR aortas. Insulin resistance blocks the inhibitor effect of etomidate on ACh-induced relaxation. Impaired $\mathrm{K}_{\text {ATP }}$ channel-mediated relaxation may be involved in the relaxation induced by etomidate in IR aortas. However, further studies are needed to confirm our preliminary conclusions.

\section{Acknowledgments}

This study was supported by the "128" Health Technology Talents Cultivation Foundation of Meitan General Hospital.

\section{Conflict of interest}

The authors declare no conflict of interest.

\section{References}

1. Romanko OP, Stepp DW. Reduced constrictor reactivity balances impaired vasodilation in the mesenteric circulation of the obese Zucker rat. Am J Physiol Heart Circ Physiol 2005; 289: H2097-2102.

2. Papaetis GS, Papakyriakou P, Panagiotou TN. Central obesity, type 2 diabetes and insulin: exploring a pathway full of thorns. Arch Med Sci 2015; 11: 463-82.

3. Busija DW, Miller AW, Katakam P, Simandle S, Erdos B. Mechanisms of vascular dysfunctionin insulin resistance. Curr Opin Investig Drugs 2004; 5: 929-35.

4. Xue W, Zhang M, Li J, Wu D, Niu L, Liang Y. Effects of taurine on aortic rings isolated from fructose-fed insulin resistance Sprague-Dawley rat are changed. Cardiovasc Drugs Ther 2008; 22: 461-8.

5. Miller AW, Katakam PV, Ujhelyi MR. Impaired endothelium-mediated relaxation in coronary arteries from insulin-resistant rats. J Vasc Res 1999; 36: 385-92.

6. Katakam PV, Domoki F, Snipes JA, Busija AR, Jarajapu YP, Busija DW. Impaired mitochondria-dependent vasodilation in cerebral arteries of Zucker obese rats with insulin resistance. Am J Physiol Regul Integr Comp Physiol 2009; 296: R289-98.

7. Kang H. Current therapeutic agents and anesthetic considerations for diabetes mellitus. Korean J Anesthesiol 2012; 63: 195-202.

8. Forman SA. Clinical and molecular pharmacology of etomidate. Anesthesiology 2011; 114: 695-707.

9. Ouedraogo N, Mounkaila B, Crevel H, Marthan R, Roux E. Effect of propofol and etomidate on normoxic and chronically hypoxic pulmonary artery. BMC Anesthesiol 2006; 6: 2.

10. Sohn JT, Murray PA. Inhibitory effects of etomidate and ketamine on adenosine triphosphate-sensitive potassium channel relaxation in canine pulmonary artery. Anesthesiology 2003; 98: 104-13.

11. Gage MC, Yuldasheva NY, Viswambharan H, et al. Endothelium-specific insulin resistance leads to accelerated atherosclerosis in areas with disturbed flow patterns: a role for reactive oxygen species. Atherosclerosis 2013; 230: 131-9.
12. Li Q, Park K, Li C, et al. Induction of vascular insulin resistance and endothelin-1 expression and acceleration of atherosclerosis by the overexpression of protein kinase C-beta isoform in the endothelium. Circ Res 2013; 113: 418-27.

13. Hsueh WA, Lyon CJ, Quinones MJ. Insulin resistance and the endothelium. Am J Med 2004; 117: 109-17.

14. Bornfeldt KE, Tabas I. Insulin resistance, hyperglycemia, and atherosclerosis. Cell Metab 2011; 14: 575-85.

15. Verma S, Bhanot S, Yao L, McNeill JH. Vascular insulin resistance in fructose-hypertensive rats. Eur J Pharmacol 1997; 322: R1-2.

16. Essawy SS, Abdel-Sater KA, Elbaz AA. Comparing the effects of inorganic nitrate and allopurinol in renovascular complications of metabolic syndrome in rats: role of nitric oxide and uric acid. Arch Med Sci 2014; 10: 537-45.

17. Shin IW, Sohn JT, Kim HJ, et al. Etomidate attenuates phenylephrine-induced contraction in isolated rat aorta. Can J Anaesth 2005; 52: 927-34.

18. Sohn JT, Kim HJ, Cho HC, Shin IW, Lee HK, Chung YK. Effect of etomidate on endothelium-dependent relaxation induced by acetylcholine in rat aorta. Anaesth Intensive Care 2004; 32: 476-81.

19. Zhang W, Zheng Y, Liu F, Wang X, Jin Z, Zhi J. Mechanism of agonistic angiotensin II type I receptor autoantibody-amplified contractile response to Ang II in the isolated rat thoracic aorta. Acta Biochim Biophys Sin (Shanghai) 2015; 47: 851-6.

20. Seymour VA, Curmi JP, Howitt SM, Casarotto MG, Laver DR, Tierney ML. Selective modulation of different GABAA receptor isoforms by diazepam and etomidate in hippocampal neurons. Int J Biochem Cell Biol 2012; 44: 1491-500.

21. Kessler P, Lischke V, Hecker M. Etomidate and thiopental inhibit the release of endothelium-derived hyperpolarizing factor in the human renal artery. Anesthesiology 1996; 84: 1485-8.

22. Boillot A, Laurant P, Berthelot A, Barale F. Effects of propofol on vascular reactivity in isolated aortae from normotensive and spontaneously hypertensive rats. $\mathrm{Br}$ J Anaesth 1999; 83: 622-9.

23. Doenicke A, Loffler B, Kugler J, Suttmann H, Grote B. Plasma concentration and E.E.G. after various regimens of etomidate. Br J Anaesth 1982; 54: 393-400.

24. Hebron BS. Plasma concentrations of etomidate during an intravenous infusion over 48 hours. Anaesthesia 1983; 38 Suppl: 39-43.

25. Doursout MF, Joseph PM, Liang YY, Hartley CJ, Chelly JE. Role of propofol and its solvent, intralipid, in nitric oxide-induced peripheral vasodilatation in dogs. $\mathrm{Br} J \mathrm{An}$ aesth 2002; 89: 492-8.

26. Lieder HR, Baars T, Kahlert P, Kleinbongard P. Aspirate from human stented saphenous vein grafts induces epicardial coronary vasoconstriction and impairs perfusion and left ventricular function in rat bioassay hearts with pharmacologically induced endothelial dysfunction. Physiol Rep 2016; 4: pii: e12874.

27. Ergil J, Kertmen H, Sayin M, et al. Vasoconstrictive effects of levobupivacaine on the basilar artery in the rabbit. Arch Med Sci 2015; 11: 654-9.

28. Palmer RM, Ashton DS, Moncada S. Vascular endothelial cells synthesize nitric oxide from L-arginine. Nature 1988; 333: 664-6.

29. Campbell WB, Harder DR. Endothelium-derived hyperpolarizing factors and vascular cytochrome P450 metabolites of arachidonic acid in the regulation of tone. Circ Res 1999; 84: 484-8. 
30. Yada T, Shimokawa H, Hiramatsu O, et al. Hydrogen peroxide, an endogenous endothelium-derived hyperpolarizing factor, plays an important role in coronary autoregulation in vivo. Circulation 2003; 107: 1040-5.

31. Gragasin FS, Davidge ST. The effects of propofol on vascular function in mesenteric arteries of the aging rat. Am J Physiol Heart Circ Physiol 2009; 297: H466-4.

32. Edwards G, Dora KA, Gardener MJ, Garland CJ, Weston $\mathrm{AH} . \mathrm{K}+$ is an endothelium-derived hyperpolarizing factor in rat arteries. Nature 1998; 396: 269-72.

33. Cersosimo E, DeFronzo RA. Insulin resistance and endothelial dysfunction: the road map to cardiovascular diseases. Diabetes Metab Res Rev 2006; 22: 423-36.

34. Ogawa K, Tanaka S, Murray PA. Propofol potentiates phenylephrine-induced contraction via cyclooxygenase inhibition in pulmonary artery smooth muscle. Anesthesiology 2001; 94: 833-9.

35. Horibe M, Ogawa K, Sohn JT, Murray PA. Propofol attenuates acetylcholine-induced pulmonary vasorelaxation: role of nitric oxide and endothelium-derived hyperpolarizing factors. Anesthesiology 2000; 93: 447-55.

36. Ludmer PL, Selwyn AP, Shook TL, et al. Paradoxical vasoconstriction induced by acetylcholine in atherosclerotic coronary arteries. N Engl J Med 1986; 315: 1046-51.

37. Oyama N, Urasawa K, Sakai H, Fujii S, Kitabatake A. Insulin resistance relates to acetylcholine-induced microvascular vasoconstriction in a patient with vasospastic angina: potential implication of causalities between hyperinsulinemia and coronary microcirculation failure. Heart Vessels 2005; 20: 72-6.

38. Katakam PV, Ujhelyi MR, Miller AW. EDHF-mediated relaxation is impaired in fructose-fed rats. J Cardiovasc Pharmacol 1999; 34: 461-7.

39. Kleinbongard P, Schleiger A, Heusch G. Characterization of vasomotor responses in different vascular territories of C57BL/6J mice. Exp Biol Med (Maywood) 2013; 238 1180-91. 